\section{Original Article}

\author{
Corresponding Author \\ Jin Hoon Park \\ (iD https://orcid.org/0000-0002-0903-3146 \\ Department of Neurosurgery, University \\ of Ulsan College of Medicine, Asan \\ Medical Center, 88 Olimpic-ro 43-gil, \\ Songpa-gu, Seoul 05505, Korea \\ Email: jhpark@amc.seoul.kr
}

Received: October 27, 2020

Revised: November 27, 2020

Accepted: December 10, 2020

See the commentary "Intraoperative

Monitoring for Cauda Equina Tumors:

Surgical Outcomes and

Neurophysiological Data Accrued Over 10

Years" via https://doi.org/10.14245/

ns.2142504.252

\begin{abstract}
(c) (1) (9)
This is an Open Access article distributed under the terms of the Creative Commons Attribution Non-Commercial License (https://creativecommons.org/licenses/by-nc/4.0/) which permits unrestricted non-commercial use, distribution, and reproduction in any medium, provided the original work is properly cited.
\end{abstract}

Copyright $\odot 2021$ by the Korean Spinal Neurosurgery Society

\title{
Intraoperative Monitoring for Cauda Equina Tumors: Surgical Outcomes and Neurophysiological Data Accrued Over 10 Years
}

\author{
Subum Lee ${ }^{1}$, Dae-Chul Cho ${ }^{1}$, Seung Chul Rhim², Byung Jou Lee ${ }^{3}$, Seok Ho Hong ${ }^{2}$, \\ Yong Seo $\mathrm{Koo}^{4}$, Jin Hoon Park ${ }^{2}$ \\ ${ }^{1}$ Department of Neurosurgery, Kyungpook National University Hospital, Kyungpook National University \\ School of Medicine, Daegu, Korea \\ ${ }^{2}$ Department of Neurosurgery, Asan Medical Center, University of Ulsan College of Medicine, Seoul, Korea \\ ${ }^{3}$ Department of Neurosurgery, Inje University Ilsan Paik Hospital, Neuroscience \& Radiosurgery Hybrid \\ Research Center, College of Medicine, Goyang, Korea \\ ${ }^{4}$ Department of Neurology, Asan Medical Center, University of Ulsan College of Medicine, Seoul, Korea
}

Objective: Cauda equina tumors affect the peripheral nervous system, and the validities of triggered electromyogram (tEMG) and intraoperative neurophysiologic monitoring (IOM) are unclear. We sought to evaluate the accuracy and relevance of tEMG combined with IOM during cauda equina tumor resection.

Methods: Between 2008 and 2018, an experienced surgeon performed cauda equina tumor resections using tEMG at a single institution. A cauda equina tumor was defined as an intradural-extramedullary or intradural-extradural tumor at the level of L2 or lower. The clinical presentation, extent of resection, pathology, recurrence, postoperative neurological outcomes, and intraoperative tEMG mapping and IOM data were retrospectively analyzed. Results: One hundred three patients who underwent intraoperative tEMG were included; 38 underwent only tEMG (tEMG-only group), and 65 underwent a combination of tEMG and multimodal IOM (MIOM group). There were no significant differences between the neurologic outcomes, extents of resection, or recurrence rates of the 2 groups. No significant therapeutic benefit was observed; however, the accuracy of intraoperative predetection improved with the combination of IOM and tEMG (accuracy: tEMG-only group, 86.8\%; MIOM group, 92.3\%). When the involved rootlet was resected despite the positive tEMG result, motor function worsened in 3 of 8 cases. The sensitivity and specificity of tEMG were $37.5 \%$ and $94.7 \%$, respectively.

Conclusion: tEMG is an essential adjunctive surgical tool for deciding on and planning for rootlet resection. If the tEMG finding is negative, complete resection, involving the rootlet, may be safe. The accuracy may be further improved by using a combination of tEMG and IOM.

Keywords: Cauda equina, Electromyogram, Neurological outcome, Neuromonitoring, Spinal cord tumors

\section{INTRODUCTION}

It is well-known that multimodal intraoperative monitoring (IOM) during spinal cord surgery is a reliable and valid diagnostic adjunct for assessing spinal cord integrity. ${ }^{1} \mathrm{IOM}$ is im- portant for preserving neuronal structures and achieving an optimal postoperative functional outcome. ${ }^{2}$ Thus, IOM has become the standard for spinal cord tumor surgery.

However, somatosensory evoked potential (SSEP) and motor evoked potential (MEP) monitoring are not routinely used dur- 
ing the resection of tumors of the lumbar spine. ${ }^{3}$ In addition, the utility of IOM for intradural-extramedullary (IDEM) tumors has not been confirmed. ${ }^{4,5}$ Tumors of the cauda equina are rare, and they account for $5 \%$ of all primary intraspinal tumors. ${ }^{6}$ To the best of our knowledge, the exclusive use of triggered electromyogram (tEMG) and other IOM modalities for cauda equina level tumors have not been the focus of previously published studies. Although root mapping using tEMG is generally performed during cauda equina surgery, ${ }^{7}$ no studies have analyzed its accuracy for cauda equina intradural tumor removal. In literature, a limited number of reports describe the intraoperative use of tEMG for tumor removal. ${ }^{8,9}$ These reports only describe the usefulness of tEMG for predicting postoperative outcomes of nerve root sacrifice in cervical spinal tumors. Furthermore, since cauda equina tumors affect the peripheral nervous system, the validity of multimodal IOM is also unclear.

We aimed to establish the usefulness of tEMG and IOM for determining surgical strategies and balancing the conflict between the following 2 objectives: (1) the complete resection of pathologic tissue and (2) the preservation of neurologic function. For this purpose, we evaluated the accuracy and relevance of monitoring surgical outcomes with tEMG mapping combined with SSEP, MEP, and bulbocavernosus reflex (BCR) monitoring during the resection of cauda equina IDEM tumors.

\section{MATERIALS AND METHODS}

\section{Patient Population and Data Selection}

Data of 127 consecutive patients who presented with cauda equina tumors treated by a single senior surgeon at a single institution between 2008 and 2018 were prospectively collected in a database and retrospectively analyzed. Cauda equina tumors in this study were defined as IDEM and intradural-extradural (IDED) tumors at the level of L2 or lower. Of these, intramedullary tumors originating from the conus medullaris or metastatic tumors were excluded. No distinction was made between different histopathological diagnoses. The patients who were not monitored using EMG or other modalities were subsequently excluded, leaving 24 patients in the final series. Spontaneous (free-running) EMG (sEMG) data were not analyzed, but tEMG reports with records on the preservation of the adjacent rootlet were analyzed. Baseline characteristics, including sex, age, IOM data, and neurological status on admission, discharge, and at 6-month follow-up, were collected. The pathological diagnosis and recurrence were also investigated. Based on the postoperative magnetic resonance imaging, recurrence was defined as an increase in the residual tumor size or the development of new lesions. This retrospective study was approved by the Institutional Review Board (IRB) of Asan Medical Center (AMC IRB 2019-1308).

\section{Intraoperative Neurophysiologic Monitoring}

Neurophysiologic monitoring was performed throughout the surgery. The baseline readings were obtained before skin incision and after the exposure of the dura mater. The stimulation alternated between SSEP and MEP. SSEP amplitude reduction of $>50 \%$ of the baseline value and latency increase by $>10 \%$ were regarded as significant. ${ }^{10-12}$ During the propofol maintenance of anesthesia, an MEP amplitude decrement of $>50 \%$ of the baseline value was considered indicative of a significant change provided that the levels of neuromuscular blockade and general anesthesia were unchanged. ${ }^{10,12,13}$ If the amplitude of the BCR fell below $50 \%$ of the baseline value after application of the above criteria, it was considered a positive sign.

In this study, motor root mapping using tEMG was regarded as a modality of IOM. A positive tEMG finding was determined as follows: after the dissection of the rootlets surrounding the tumor, the nerve that was considered as the origin of the tumor was separated with a hook and stimulated with a bipolar nerve stimulator (current: $3-10 \mathrm{~mA}$ ). ${ }^{14}$ To reduce the false positives, a cottonoid was used to insulate the dissected rootlets (Fig. 1). A recording of all the lumbosacral myotomes (sphincter ani exter-

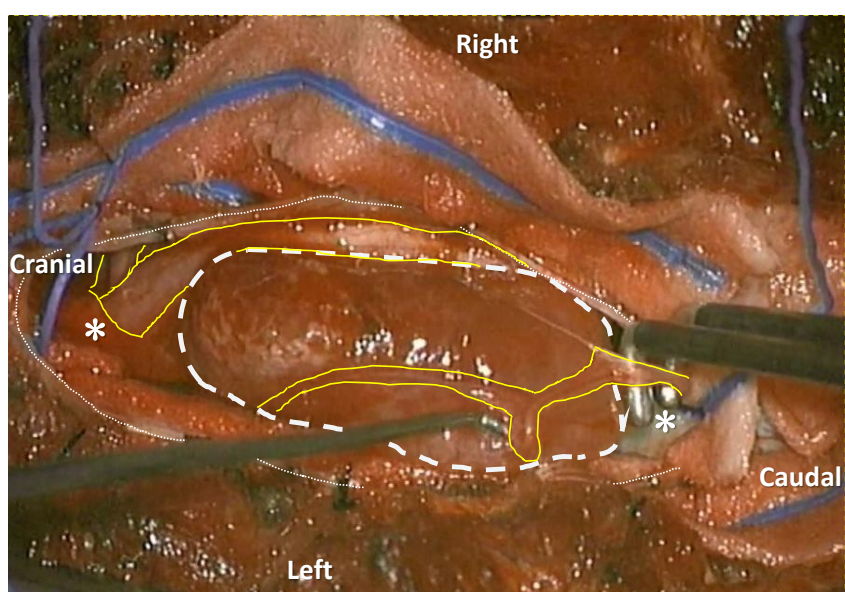

Fig. 1. Triggered electromyogram (tEMG) method: meticulous dissection of the rootlet to preserve nerve function. A right-angle hook was used to pull the rootlet away from the surrounding tissue, including the tumor. After minimizing current interference with a cotton pattie, tEMG was performed using a bipolar stimulator (white dashed line: tumor; white dotted line: dura; yellow full line: rootlet; asterisk: cotton pattie). 
nus, abductor hallucis, gastrocnemius, iliopsoas, tibialis anterior, and vastus lateralis) from all the representative segmental target muscles ensured that all possibly affected motor roots were covered. ${ }^{7}$ After stimulating and confirming the compound muscle action potential in the corresponding muscles, a positive finding was characterized by a complete resection of the root, whereas a negative finding was characterized by the preservation of some involved rootlets, even with the tEMG signal.

Table 1. Patient demographics and primary surgical outcomes of both groups

\begin{tabular}{|c|c|c|c|c|}
\hline Variable & tEMG-only $(\mathrm{n}=38)$ & $\operatorname{MIOM}(\mathrm{n}=65)$ & p-value & Total \\
\hline Age (yr) & $46.0 \pm 15.5$ & $45.0 \pm 15.4$ & 0.752 & $45.4 \pm 15.3$ \\
\hline Sex & & & 0.804 & \\
\hline Male & $16(42.1)$ & $29(44.6)$ & & 45 (43.7) \\
\hline Female & $22(57.9)$ & $36(55.4)$ & & $58(56.3)$ \\
\hline \multicolumn{5}{|l|}{ Follow-up period (mo) } \\
\hline $\mathrm{MR}$ & $30.6 \pm 31.6$ & $14.8 \pm 16.2$ & 0.006 & $20.6 \pm 24.2$ \\
\hline Clinical & $35.9 \pm 27.9$ & $17.4 \pm 14.7$ & 0.001 & $24.2 \pm 22.3$ \\
\hline Tumor location & & & 0.786 & \\
\hline Intradural-extradural & $4(10.5)$ & $8(12.3)$ & & $12(11.7)$ \\
\hline Intradural only & $34(89.5)$ & $57(87.7)$ & & $91(88.3)$ \\
\hline Lesion level & & & 0.784 & \\
\hline Multilevel lesion & $5(13.2)$ & $10(15.4)$ & & $15(14.6)$ \\
\hline Single level lesion & $33(86.8)$ & $55(84.6)$ & & $88(85.4)$ \\
\hline \multicolumn{5}{|l|}{ Pathologic diagnosis } \\
\hline Schwannoma & $32(84.2)$ & $53(81.5)$ & 0.794 & $85(82.5)$ \\
\hline Myxopapillary ependymoma & $3(7.9)$ & $4(6.2)$ & 0.707 & $7(6.8)$ \\
\hline Meningioma & $2(5.3)$ & $0(0)$ & 0.134 & $2(1.9)$ \\
\hline Others $^{*}$ & $1(2.6)$ & $8(12.3)$ & 0.094 & $9(8.7)$ \\
\hline \multicolumn{5}{|l|}{ Preoperative neurological deficit } \\
\hline Overall & $3(7.9)$ & $8(12.3)$ & 0.488 & $11(10.7)$ \\
\hline Weakness & $\begin{array}{c}2 \text { (5.3); } 1 \text { unilateral, } \\
1 \text { bilateral }\end{array}$ & $\begin{array}{c}4 \text { (6.2); } 2 \text { unilateral, } \\
2 \text { bilateral }\end{array}$ & 0.852 & $6(5.8)$ \\
\hline Hypoesthesia (saddle anesthesia) & $1(2.6)$ & $3(4.6)$ & 0.613 & $4(3.9)$ \\
\hline Bladder-bowel symptom & $1(2.6)$ & $1(1.5)$ & 0.695 & $2(1.9)$ \\
\hline \multicolumn{5}{|l|}{ Extent of tumor resection } \\
\hline Gross total resection & $31(81.6)$ & $49(75.4)$ & 0.625 & $80 / 103(77.7)$ \\
\hline Subtotal $(>50)$ resection & $7(18.4)$ & $16(24.6)$ & 0.468 & $23 / 103(22.3)$ \\
\hline \multicolumn{5}{|l|}{ Recurrence } \\
\hline Overall & $5(13.2)$ & $11(16.9)$ & 0.780 & $16 / 103(15.5)$ \\
\hline Gross total resection & $1 / 31(3.2)$ & $2 / 49(4.1)$ & 0.893 & $3 / 80(3.8)$ \\
\hline Subtotal $(>50)$ resection & $4 / 7(57.1)$ & $9 / 16(56.2)$ & 0.969 & $13 / 23(56.5)$ \\
\hline \multicolumn{5}{|c|}{ Postoperative neurological deterioration } \\
\hline Overall & $12(31.6)$ & $12(18.5)$ & 0.131 & $24 / 103(23.3)$ \\
\hline Motor & $4(10.5)$ & $4(6.2)$ & 0.463 & 8/103 (7.8) \\
\hline Sensory & $8(21.1)$ & $8(12.3)$ & 0.268 & $16 / 103(15.5)$ \\
\hline
\end{tabular}

Values are presented as mean \pm standard deviation or number (\%).

tEMG, triggered electromyogram; MIOM, multimodal intraoperative monitoring (tEMG + MEP + SSEP + BCR); MEP, motor evoked potential; SSEP, somatosensory evoked potential; BCR, bulbocavernosus reflex.

${ }^{\star}$ Others pathologic diagnoses: 1 mixed germ cell tumor (tEMG-only group); 1 ependymoma; 1 epidermoid cyst; 1 Ewing sarcoma/primitive neuro-ectodermal tumor (MIOM group); 1 hemangioblastoma; 1 lobular capillary hemangioma; 1 mesenchymal chondrosarcoma; 1 neurofibroma; 1 paraganglioma. 


\section{Postoperative Neurologic Deficits}

The neurological state of each patient was evaluated before and immediately after surgery and 6 months later or after a more extended period in the outpatient clinics. Neurological deterioration was defined as new-onset permanent weakness, hypoesthesia, bladder-bowel symptoms after surgery, or worsening of preoperative deficits. Although the last follow-up duration varied from patient to patient, a permanent deficit was defined as a neurologic deficit that persisted after 6 months.

All positive IOM signs were correlated with the findings of neurologic examinations performed by attending surgeons, including neurological spine fellows and residents. A true-positive IOM change was associated with a neurologic deterioration; a false-positive IOM change was not. A true-negative IOM change was associated with a postoperative neurologic deterioration; a false-negative IOM change was not.

\section{Statistical Analysis}

Sensitivity, specificity, positive predictive value, negative predictive value, prevalence, relative risk, and accuracy, including $95 \%$ confidence intervals, were calculated. The data were analyzed for the entire group and subanalyzed based on the monitoring modality. The Student t-test or Mann-Whitney U-test was used to compare continuous variables, and the Pearson chisquare test or Fisher exact test was used to compare discrete variables of the groups. We used IBM SPSS Statistics ver. 21.0 (IBM Co., Armonk, NY, USA) for statistical analyses. A p-value of $<0.05$ was considered statistically significant.

\section{RESULTS}

\section{Patient Demographics and Clinical Presentation}

A summary of the demographic data is provided in Table 1. A total of 103 patients who underwent cauda equina tumor resection with IOM were included. Of these, 38 underwent tEMG only (tEMG-only group), and 65 underwent multimodal IOM (MIOM group; tEMG, MEP, SSEP, and BCR combined). The

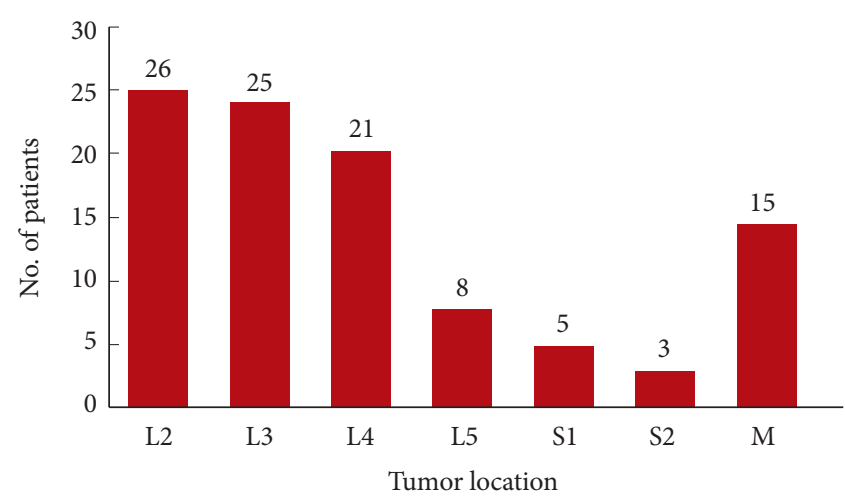

Fig. 2. Distribution of cauda equina tumors in each segment for the 103 cases. M, multilevel lesion.
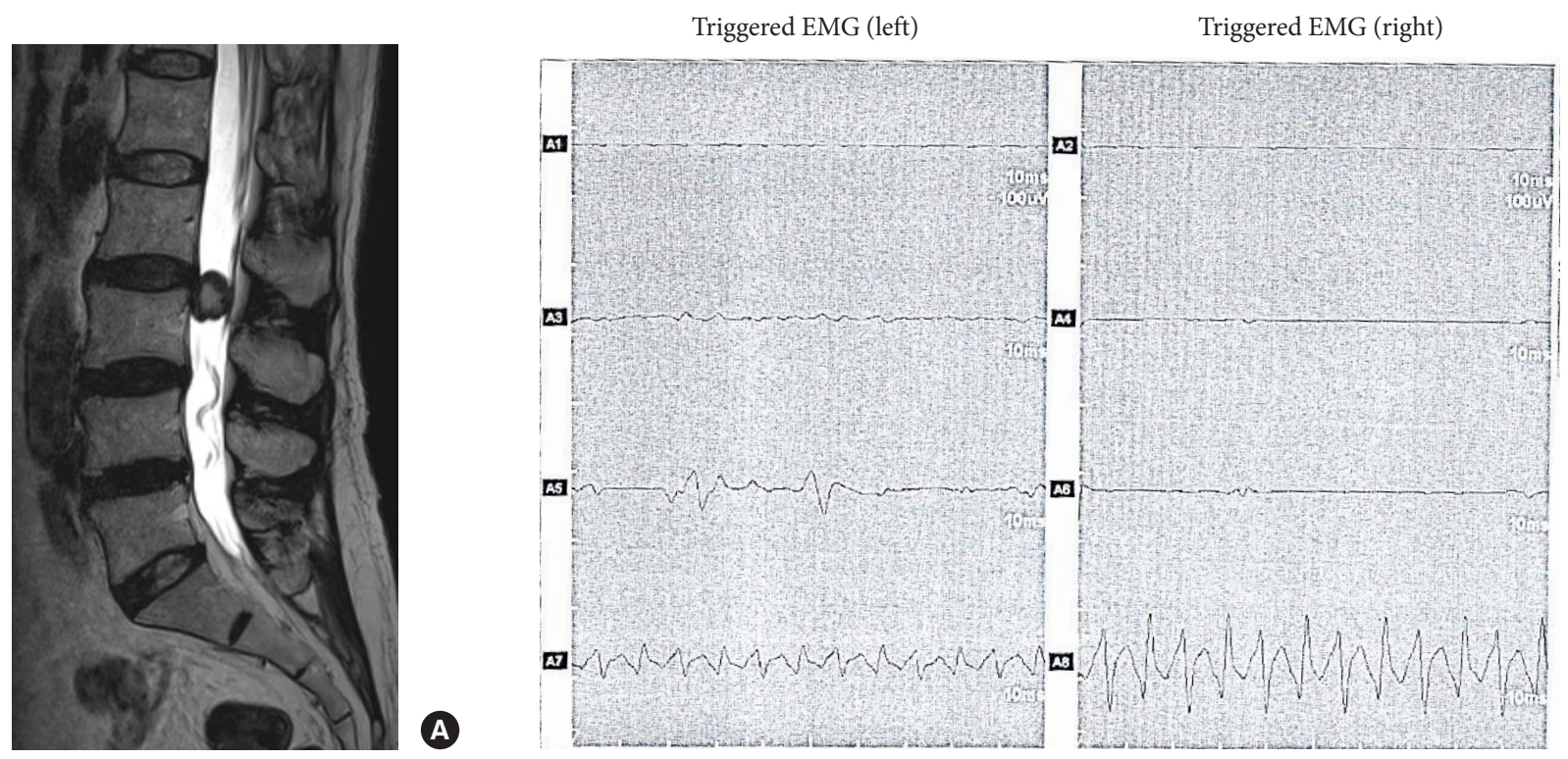

B

Fig. 3. (A) Preoperative magnetic resonance imaging of a 60-year-old woman who presented with radiating pain in the left leg shows a round intradural-extramedullary mass at the L3 level. (B) On intraoperative triggered electromyogram (EMG), action potentials were identified for the anus bilaterally (A7 \& A8) and the left gastrocnemius (A5). A1 \& A2, left and right vastus lateralis; A3 \& A4, left and right tibialis anterior; A5 \& 6, left and right gastrocnemius; A7 \& 8, left and right sphincter ani externus. 
mean age of the population was $45.4 \pm 15.3$ years; 45 (43.7\%) were males and 58 (56.3\%) were females. All patients underwent surgery with the posterior approach and laminoplasty. IDEM tumors were resected in 91 patients (88.3\%), and IDED tumors were resected in 12 patients (11.7\%). Tumor size did not exceed one vertebral level in 88 patients, whereas 15 patients had multiple or multilevel lesions (Fig. 2). The most common diagnosis was schwannoma in 85 patients (82.5\%), followed by myxopapillary ependymoma in 7 (6.8\%), and meningioma in 2 (1.9\%). Nine patients were diagnosed with other tumors (1 ependymoma, 1 epidermoid cyst, 1 Ewing sarcoma/primitive neuro-ectodermal tumor, 1 hemangioblastoma, 1 lobular capillary hemangioma, 1 mesenchymal chondrosarcoma, 1 mixed germ cell tumor, 1 neurofibroma, 1 paraganglioma).

The clinical presentations of the patients are as follows: the most common chief complaint was pain in 94 of 103 patients (91.3\%). Four of 103 patients (3.9\%) had intermittent claudication, $1(1.0 \%)$ had weakness, $1(1.0 \%)$ had urinary symptoms, and $3(2.0 \%)$ had an incidental finding. Preoperative neurologic examination on admission revealed that 6 patients (5.8\%) had objective weakness (unilateral lower limb: 3 , bilateral lower limb: 3), 4 (3.9\%) had hypoesthesia, including saddle anesthesia, and $2(1.9 \%)$ had bladder or bowel symptoms. There was no significant difference between the tEMG-only and MIOM groups, except for the clinical follow-up duration (Table 1).

\section{Postoperative Outcome}

Regarding the chief complaint, 72 of 94 patients (76.6\%) who complained of pain experienced relief. Regarding the neurological outcomes, a permanent postoperative motor deterioration, including bladder and/or bowel symptoms, was observed in 8 of 103 patients (7.8\%). Two patients ( 2 of 103, 1.9\%) had bladder and/or bowel symptoms and 6 (6 of 103, 5.8\%) had skeletal muscle weakness in a lower limb; the other 16 of 103 patients (15.5\%) had sensory deficits. There was no significant difference between the incidences of postoperative neurologic dete-

Table 2. Crosstables of true positives, false positives, true negatives, and false negatives

\begin{tabular}{|c|c|c|c|}
\hline \multicolumn{4}{|c|}{ Overall (tEMG-only group + MIOM group, $\mathrm{n}=103$ ) } \\
\hline tEMG - overall & Neurological deficit $(+)$ & Neurological deficit (-) & Subtotal \\
\hline EMG $(+) \&$ root sacrifice & 3 & 5 & 8 \\
\hline EMG (-) or (EMG [+] \& root preserve) & 5 & 90 & 95 \\
\hline Subtotal & 8 & 95 & 103 \\
\hline \multicolumn{4}{|c|}{ tEMG-only group $(\mathrm{n}=38)$} \\
\hline tEMG single & Neurological deficit $(+)$ & Neurological deficit (-) & Subtotal \\
\hline EMG $(+) \&$ root sacrifice & 3 & 4 & 7 \\
\hline EMG (-) or (EMG [+] \& root preserve) & 1 & 30 & 31 \\
\hline Subtotal & 4 & 34 & 38 \\
\hline \multicolumn{4}{|c|}{ MIOM group $(n=65)$} \\
\hline tEMG with MEP & Neurological deficit $(+)$ & Neurological deficit (-) & Subtotal \\
\hline EMG $(+) \&$ root sacrifice & 0 & 1 & 1 \\
\hline EMG (-) or (EMG [+] \& root preserve) & 4 & 60 & 64 \\
\hline Subtotal & 4 & 61 & 65 \\
\hline MEP & Neurological deficit $(+)$ & Neurological deficit (-) & Subtotal \\
\hline $\operatorname{MEP}(+)$ & 3 & 6 & 9 \\
\hline $\operatorname{MEP}(-)$ & 1 & 55 & 56 \\
\hline Subtotal & 4 & 61 & 65 \\
\hline SSEP & Neurological deficit $(+)$ & Neurological deficit (-) & Subtotal \\
\hline $\operatorname{SSEP}(+)$ & 3 & 0 & 3 \\
\hline $\operatorname{SSEP}(-)$ & 5 & 57 & 62 \\
\hline Subtotal & 8 & 57 & 65 \\
\hline
\end{tabular}

tEMG, triggered electromyogram; MIOM, multimodal intraoperative monitoring (tEMG + MEP + SSEP + BCR); MEP, motor evoked potential; SSEP, somatosensory evoked potential; BCR, bulbocavernosus reflex; N.Deficit, neurological deficit; 
rioration in the 2 groups. Similarly, there were no statistically significant differences between the gross total resection (GTR) or recurrence rates of the 2 groups. Three of the 80 patients (3.8\%) who underwent GTR surgery had recurrence; 2 had schwannomas and one had a meningioma. Of the 27 patients who underwent subtotal resections (defined as resection of more than $50 \%$ of the tumor but less than complete resection), there was recurrence in $13(56.5 \%)$ (Table 1$)$.

\section{3. tEMG and IOM Data Analysis}

Of the 103 patients with tEMG mapping, 8 had a positive tEMG (Fig. 3). Three of these 8 patients developed postoperative motor deficits (true positive) and 5 did not (false positive). The tEMG was negative in 95 patients; 5 developed postoperative motor deficits (false negative) and 90 did not (true negative). The true-positive, false-positive, true-negative, and falsenegative tEMG, MEP, and SSEP of the tEMG-only and the MIOM groups were counted. We have summarized the results in Table 2.

The overall tEMG of all the patients showed a sensitivity of $37.5 \%$, a specificity of $94.7 \%$, and an accuracy of $90.3 \%$. When only tEMG was performed, the sensitivity was $75.0 \%$, the specificity was $88.2 \%$, and the accuracy was $86.8 \%$. When combined with MEP, tEMG showed a sensitivity of $0 \%$, a specificity of $98.4 \%$, and an accuracy of $92.3 \%$. The sensitivity and specificity of MEP were $75.0 \%$ and $90.2 \%$, and the sensitivity and specificity of SSEP were $37.5 \%$ and $100.0 \%$, respectively (Table 3).

One patient in the MIOM group who underwent intraoperative BCR monitoring showed a positive sign (100\% amplitude loss) and developed defecation and urination disorders as postoperative complications. Overall, 2 patients had bladder and/or bowel symptoms after surgery; 11 had a positive sign (an amplitude reduction of less than 50\%).

\section{An Illustrative Case}

A 60-year-old woman presented with radiating pain in the left leg. The diagnosis was schwannoma (Fig. 3). On tEMG, action potentials were identified for the anus bilaterally (A7 \& A8) and the left gastrocnemius (A5); the action potential was especially definite for the right anus (A8). Before the final assessment of the involved rootlet, the tEMG finding was positive. However, the nerve rootlet was resected as part of GTR. After the surgery, the patient developed urinary incontinence (stimulation settings: rate, $5 \mathrm{~Hz}$; duration, $0.1 \mathrm{msec}$; intensity, $5.0 \mathrm{~mA})$.

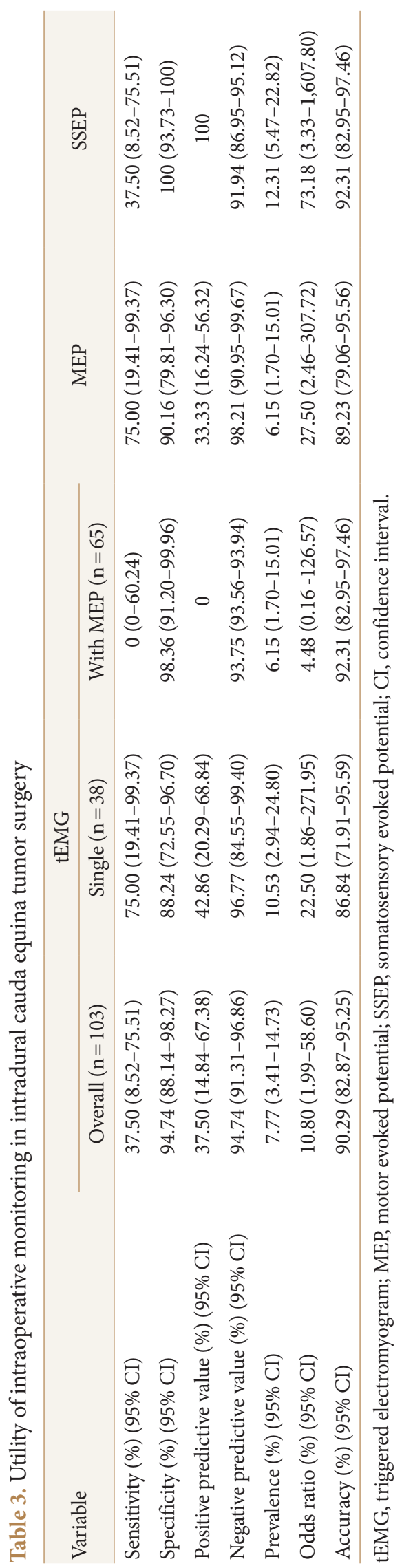




\section{DISCUSSION}

We reported the outcomes of the cauda equina tumor resection performed by a single surgeon based on 10-year consecutive data. The most common histological type within the anatomical range was schwannoma (82.5\%), followed by myxopapillary ependymoma (6.8\%). In a French multicenter retrospective review of 231 cases of adult cauda equina tumors, ${ }^{15}$ the most common histologic type was schwannoma (49.3\%), followed by ependymoma (34.9\%). This is consistent with our findings, although the proportions are different.

IDEM tumors of the cauda equina are rarely associated with postoperative neurological deficits. ${ }^{6}$ This has been proposed as a basis for objecting to the need for IOM during cauda equina tumor surgery. The goal of IDEM tumor surgery is to enable the GTR of the tumor while preserving neurological function. However, we often encounter situations in which these 2 goals conflict. A total of 80 patients underwent GTR, and they showed a recurrence rate of $3.8 \%$ ( 3 of 80 ). Conversely, 23 patients with subtotal resection had a higher recurrence rate of $56.5 \%$ (13 of 23). Based on these results, even if cauda equina tumors are mostly benign, the surgeon should make maximal efforts to achieve GTR. Neuromonitoring is important as it increases the rates of total resection while facilitating the preservation of neurological function. ${ }^{16}$ In contrast, there was no preventive role for multimodal IOM in cauda equina tumor removal in our study. The surgical outcomes showed no significant differences in neurologic deficits, GTR, or recurrence rate in patients in the tEMG-only and MIOM groups (Table 1). These results are consistent with recent guidelines by Hadley et al. ${ }^{17}$ for patients undergoing spinal cord intramedullary tumor resection. There is class I medical evidence supporting the value of IOM as a diagnostic tool for assessing spinal cord integrity in the perioperative setting. ${ }^{1718}$ In lumbosacral spinal procedures, the preservation-related focus of neurological function shifts to the nerve root level, as only the thecal sac and nerve roots are located below the conus medullaris. ${ }^{19}$

Since there was no significant difference between the clinical outcomes of the 2 groups, we focused on the validity of the clinical application of tEMG for cauda equina tumor surgery. tEMG could help determine whether the surgeon will eventually leave residual tumor to preserve the rootlet or resect the rootlet and achieve GTR. In other words, tEMG guides the final decision on sacrificing the nerve, whereas MEP and SSEP are useful for assessing whether the patient will develop neurologic function deficits after the nerve sacrifice. Although MEP and SSEP can be used to diagnose intraoperative neurological injuries, their advantages related to preventing new neurological deficits after spinal surgery are uncertain. ${ }^{18}$

The sensitivity of tEMG was lower (37.5\%) than that previously reported, ${ }^{20}$ because we included only lesions at the level of the cauda equina. tEMG monitors only a single rootlet, while 2 or 3 nerve segment innervations underlie the final key muscle function based on the results of electrical stimulation studies. ${ }^{21,22}$ An additional hypothesis is that a gradual loss of function of the affected root is compensated by concomitant reinnervation of the dependent peripheral structures via the nerve endings of the other roots. ${ }^{23}$ When the nerve bundles of the index root are compressed by tumor growth and the lesion of the root develops slowly, functional compensation by innervation from neighboring roots may gradually become predominant. ${ }^{24}$ Meanwhile, it is important to perform meticulous dissection of the involved nerve fiber to maximize specificity by gently pulling it away from other surrounding rootlets and tumors. In this procedure, a cotton pattie should be placed under the involved nerve fiber before stimulation to achieve complete isolation, because current interference through other rootlets during stimulation using bipolar forceps leads to false-positive findings (Fig. 1).

Paradiso et al.$^{20}$ suggested that SSEP in combination with sEMG is the optimal choice for monitoring in tethered cord release surgery. In that study, the prognostic values of the modalities were similar to those of other lumbosacral procedures owing to the high specificity and relatively low sensitivity of SSEP, which was complemented by a sensitivity of $100 \%$ for sEMG/tEMG. Similarly, SSEP showed high specificity (100\%) in our study. Although the most common neurological deficit after cauda equina tumor surgery was hypoesthesia, the clinical validity of SSEP was very low because it is very limited in assessing sensory deficits within the global dermatomes of the lower limb; the posterior tibial nerve was the only site being monitored. Nevertheless, SSEP can be used to continuously monitor the sensory pathways of L5 and S1.

Theoretically, BCR examination is effective for functional monitoring of the genitalia, anus, and urethral sphincter. In this study, one of the 65 patients who underwent intraoperative BCR monitoring showed a positive sign ( $>50 \%$ amplitude loss on bilateral BCR monitoring) and developed a severe defecation disorder postoperatively. Because there was only 1 case with serious complications, BCR monitoring showed high specificity, but the finding was not statistically significant. The overall incidence of bladder and/or bowel symptoms after cauda equina IDEM tumor surgery was low (2 of 103, 1.9\%). Nevertheless, 
the preservation of the S2-4 roots and the pudendal nerve function has the most substantial influence on the quality of life of patients. We believe that changing the criteria to below 50\% will be helpful; however, this will require further validation.

This study has several potential limitations. First, there was no histological distinction during the outcome analysis. However, surgeons are usually unsure of the exact histology of the tumor before surgery, and they prepare for perioperative monitoring based on the differential diagnosis of IDEM tumors. Therefore, various histological types were included in the study to evaluate the clinical usefulness of IOM in practice. Second, we did not perform a correlation analysis for sEMG. Third, the MEP, SSEP, and BCR outcome measurement definitions based on the $<50 \%$ threshold were not optimum; perhaps those thresholds were not adequate, given that they were reported for intramedullary spinal cord tumors. Fourth, since all the surgeries were performed by a single surgeon, technical progress over 10 years may have affected the results. The surgeries were performed with tEMG alone earlier and multimodal IOM later in this study, and this may have affected the clinical results. Finally, this study was limited by its retrospective design.

\section{CONCLUSION}

tEMG is useful for determining whether the involved rootlet needs to be resected. Overall, tEMG showed a low sensitivity $(37.5 \%)$ and a high specificity (94.7\%). Thus, when the tEMG finding is negative, it may be safe to perform the surgery without nerve preservation. Even if the tEMG finding is positive, the probability of actual motor deterioration is less than $50 \%$. The specificity and accuracy can be further improved by using a combination of tEMG and IOM. Nevertheless, to minimize permanent deterioration, maximal efforts should be put into preserving the rootlet through meticulous dissection. All IOM modalities, including tEMG, could not prevent false negatives.

\section{CONFLICT OF INTEREST}

The authors have nothing to disclose.

\section{REFERENCES}

1. Fehlings MG, Brodke DS, Norvell DC, et al. The evidence for intraoperative neurophysiological monitoring in spine surgery: does it make a difference? Spine 2010;35:S37-46.

2. Scibilia A, Terranova C, Rizzo V, et al. Intraoperative neuro- physiological mapping and monitoring in spinal tumor surgery: sirens or indispensable tools? Neurosurgical focus 2016; 41:E18.

3. Lakomkin N, Mistry AM, Zuckerman SL, et al. Utility of intraoperative monitoring in the resection of spinal cord tumors: an analysis by tumor location and anatomical region. Spine 2018;43:287-94.

4. Costa P, Peretta P, Faccani G. Relevance of intraoperative D wave in spine and spinal cord surgeries. Eur Spine J 2013; 22:840-8.

5. Ghadirpour R, Nasi D, Iaccarino C, et al. Intraoperative neurophysiological monitoring for intradural extramedullary tumors: why not? Clin Neurol Neurosurg 2015;130:140-9.

6. Engelhard HH, Villano JL, Porter KR, et al. Clinical presentation, histology, and treatment in 430 patients with primary tumors of the spinal cord, spinal meninges, or cauda equina. J Neurosurg Spine 2010;13:67-77.

7. Kothbauer KF, Deletis V. Intraoperative neurophysiology of the conus medullaris and cauda equina. Childs Nerv Syst 2010;26:411-2.

8. Guo L, Quiñones-Hinojosa A, Yingling CD, et al. Continuous EMG recordings and intraoperative electrical stimulation for identification and protection of cervical nerve roots during foraminal tumor surgery. J Spinal Disord Tech 2006; 19:37-42.

9. Kaneko K, Kato Y, Kojima T, et al. Intraoperative electrophysiologic studies on the functions of nerve roots involved in cervical dumbbell-shaped schwannoma and their clinical utility. J Spinal Disord Tech 2006;19:571-6.

10. Burke D, Nuwer MR, Daube J, et al. Intraoperative monitoring. The International Federation of Clinical Neurophysiology. Electroencephalogr Clin Neurophysiol Suppl 1999;52: 133-48.

11. Choi I, Hyun SJ, Kang JK, et al. Combined muscle motor and somatosensory evoked potentials for intramedullary spinal cord tumour surgery. Yonsei Med J 2014;55:1063-71.

12. Hyun SJ, Rhim SC. Combined motor and somatosensory evoked potential monitoring for intramedullary spinal cord tumor surgery: correlation of clinical and neurophysiological data in 17 consecutive procedures. Br J Neurosurg 2009; 23:393-400.

13. Pelosi L, Lamb J, Grevitt M, et al. Combined monitoring of motor and somatosensory evoked potentials in orthopaedic spinal surgery. Clin Neurophysiol 2002;113:1082-91.

14. Holland NR. Intraoperative electromyography during thoracolumbar spinal surgery. Spine 1998;23:1915-22. 
15. Wager M, Lapierre F, Blanc JL, et al. Cauda equina tumors: a French multicenter retrospective review of 231 adult cases and review of the literature. Neurosurg Rev 2000;23:119-29; discussion 30-1.

16. Tessitore E, Cabrilo I, Boex C, et al. Cauda equina tumor surgery: how I do it. Acta Neurochir (Wien) 2012;154:181520.

17. Hadley MN, Shank CD, Rozzelle CJ, et al. Guidelines for the use of electrophysiological monitoring for surgery of the human spinal column and spinal cord. Neurosurgery 2017;81: 713-32.

18. Daniel JW, Botelho RV, Milano JB, et al. Intraoperative neurophysiological monitoring in spine surgery: a systematic review and meta-analysis. Spine 2018;43:1154-60.

19. Gonzalez AA, Jeyanandarajan D, Hansen C, et al. Intraoperative neurophysiological monitoring during spine surgery: a review. Neurosurg Focus 2009;27:E6.
20. Paradiso G, Lee GY, Sarjeant R, et al. Multimodality intraoperative neurophysiologic monitoring findings during surgery for adult tethered cord syndrome: analysis of a series of 44 patients with long-term follow-up. Spine 2006;31:2095102.

21. Kirshblum SC, Burns SP, Biering-Sorensen F, et al. International standards for neurological classification of spinal cord injury (revised 2011). J Spinal Cord Med 2011;34:535-46.

22. McCulloch JA, Waddell G. Variation of the lumbosacral myotomes with bony segmental anomalies. J Bone Joint Surg Br 1980;62-B:475-80.

23. Schultheiss R, Gullotta G. Resection of relevant nerve roots in surgery of spinal neurinomas without persisting neurological deficit. Acta Neurochir (Wien) 1993;122:91-6.

24. Celli P. Treatment of relevant nerve roots involved in nerve sheath tumors: removal or preservation? Neurosurgery 2002; 51:684-92; discussion 92. 